\title{
Insatisfacción con la imagen corporal y personalidad en estudiantes de bachillerato de la región Altos Sur de Jalisco, México
}

\section{Dissatisfaction with body image and personality in high school students of the region Altos Sur de Jalisco, Mexico}

Título corto: Insatisfacción con la imagen corporal y personalidad en estudiantes

\begin{abstract}
Juan Francisco Caldera-Montes ${ }^{1}$ (D), Oscar Ulises Reynoso-González ${ }^{2}$ (D), Diana Nuño-Camarena ${ }^{3}$ (D), Iván Alejandro Caldera-Zamora ${ }^{4}$ (D), Ignacio Pérez-Púlido ${ }^{5}$ (D) Claudia Arcelia Gómez-Álvarez ${ }^{6}$
\end{abstract}

Tipología: Artículo de investigación científica y tecnológica

Para citar este artículo: Caldera-Montes JF, Reynoso-González O, Nuño-Camarena D, Caldera-Zamora IA, PérezPúlido I, Gómez-Álvarez CA. Insatisfacción con la imagen corporal y personalidad en estudiantes de bachillerato de la región Altos Sur de Jalisco, México. Duazary.2019 enero; 16(1): 93-103. Doi:http://dx.doi.org/10.21676/2389783X.2534

Recibido en septiembre 14 de 2017

Aceptado en diciembre 22 de 2017

Publicado en línea en septiembre 01 de 2018

\section{RESUMEN}

El presente estudio se planteó como objetivos identificar el nivel predictivo que tienen las dimensiones de la personalidad sobre la imagen corporal (IC) y sus posibles relaciones, así como comparar dichas variables conforme al sexo de los participantes. La población de estudio fueron alumnos de tres carreras técnicas de una institución de educación pública del estado de Jalisco, México. La muestra estuvo compuesta por 567 alumnos. El instrumento para medir la imagen corporal fue el Cuestionario de Insatisfacción con la Imagen Corporal (Body Shape Questionnaire) y para evaluar la Personalidad, el Inventario de Personalidad NEO Revisado NEO FFI (versión reducida del NEO PI-R). En materia de resultados y mediante un análisis de regresión lineal múltiple se identificaron como variables predictoras de la imagen corporal —insafisfecha_la dimensión de la personalidad denominada neuroticismo y el sexo femenino; además, se encontraron relaciones significativas entre las dimensiones de la personalidad neuroticismo, extroversión, amabilidad y responsabilidad con la IC y diferencias significativas en esta última conforme al sexo.

1. Universidad de Guadalajara, Jalisco, México. Correo: jfcaldera@cualtos.udg.mx - http://orcid.org/0000-0002-8999-3736

2. Universidad de Guadalajara. Jalisco, México. Correo: oscar_jalos@hotmail.com - http://orcid.org/0000-0002-0598-4665

3. Universidad de Guadalajara. Jalisco, México. Correo: diana.lenu@outlook.es - http://orcid.org/0000-0002-7570-7620

4. Universidad de Guadalajara. Jalisco, México. Correo: ivancaldera93@hotmail.com - http://orcid.org/0000-0002-1790-7560

5. Universidad de Guadalajara. Jalisco, México. Correo: iperez@cualtos.udg.mx - http://orcid.org/0000-0002-8215-8850

6.CentrodeBachillerato Tecnológico Agropecuario.Jalisco,México.Correo:claudia10c@hotmail.com-http://orcid.org/0000-0001-5744-9325 
Se sugiere que en estudios posteriores se incluyan otras características que pudieran explicar en mayor medida la presencia de Insatisfacción de la Imagen Corporal en adolescentes y se confirme la influencia de las variables predictoras identificadas.

Palabras clave: imagen corporal; personalidad; estudiantes.

\section{ABSTRACT}

The objectives of this study were to identify the predictive level that personality dimensions have on the students Body Image, as well as their possible relationships, and to compare such variables according to the sex of the participants. The study population were students of three technical careers at a public educational institution in the state of Jalisco, Mexico. The sample consisted of 567 students. The Body Shape Questionnaire was used to measure the Body Image. The Neo Personality Inventory Revised NEO FFI (reduced version of the NEO PI-R) was used to evaluate the personality. Regarding the results, a multiple linear regression analysis identified the predictive variables of the Body Image - unsatisfied-, the dimension of the personality called "Neuroticism" and the female sex. In addition, significant relationships were found between the dimensions of personality "Neuroticism", "Extroversion", "Kindness and Responsibility" with body image and significant differences in it according to sex. It is suggested that later studies include other characteristics that could explain the presence of Body Image dissatisfaction in adolescents and confirm the influence of the predictor variables identified.

Keywords: Body image; Personality; Students.

\section{INTRODUCCIÓN}

$E^{n}$ n la actualidad se cuenta con evidencia suficiente para destacar la importancia que tiene la propia imagen corporal con la salud mental de las personas, de ahí que en los últimos años se le haya dado mucha importancia a nivel mundial a los estudios sobre la Imagen Corporal (IC) en diferentes aspectos ${ }^{1}$. En este sentido, la investigación se ha centrado en la preocupación exagerada que produce malestar respecto a la apariencia física, la insatisfacción del sujeto con la forma de su cuerpo ,o bien la insatisfacción con aquellas partes de este que más preocupan a los individuos ${ }^{2}$. Además, se ha descrito que la alteración de la percepción de la imagen corporal puede considerarse un factor de riesgo para los trastornos de conducta alimentaria ${ }^{3}$, la depresión $^{4}$ y la baja autoestima ${ }^{5}$, así como otros trastornos que pueden interferir en la vida cotidiana $\mathrm{y}$ actividades personales, en los que no solo se considera un factor de riesgo, sino también una consecuencia, como la obesidad y el sobrepeso ${ }^{6,7}$.
Algunos autores definen la Imagen Corporal como la actitud hacia la apariencia del propio cuerpo, es decir, la forma en cómo uno percibe, imagina, siente y actúa respecto del mismo ${ }^{3,8-10}$. El concepto abarca aquellas opiniones, creencias y sentimientos de una persona respecto a su propio cuerpo y aspecto físico, además, algunos investigadores la relacionan con la autoestima, que se define como la capacidad de tener confianza y respeto por sí mismo ${ }^{11}$.

Se ha sugerido que la IC está formada por diferentes componentes: el componente perceptual - percepción del cuerpo-, el componente cognitivo - valoraciones, creencias, constructos personales-, el componente afectivo - sentimientos-, y por último, el componente conductual -acciones o comportamientos que se dan a partir de la percepción- ${ }^{2,10}$. Por otra parte, otros autores han sugerido que es evidente que la IC se compone de aspectos de representación conscientes, pero que además existen otros aspectos inconscientes, entre los cuales se habla 
Juan Francisco Caldera-Montes, Oscar Ulises Reynoso-González, Diana Nuño-Camarena, Iván Alejandro Caldera-Zamora, Ignacio Pérez-Púlido, Claudia Arcelia Gómez-Álvarez

de dos dimensiones: una social y una individual, incluyendo tanto la vivencia que interpreta el individuo sobre su propio cuerpo, así como las experiencias y reacciones de otras personas ${ }^{8,12}$.

Los individuos con autopercepción de IC insatisfactoria pueden tener complicaciones y repercusiones, tanto sociales como emocionales ${ }^{2,4}$. La gran presión social a la que se ven sometidos, en particular mujeres, adolescentes y jóvenes ${ }^{13}$, con la imposición de un modelo de extrema delgadez, hace que la preocupación por la imagen corporal se haya involucrado cada vez más con el mundo de la salud física y mental y el desarrollo de trastornos como los anteriormente señalados ${ }^{1,14}$. En México, para 1997, un estudio realizado con 200 preadolescentes ya estimaba la insatisfacción con la IC en un $50 \%{ }^{15}$; para 2008 , otra investigación con 403 estudiantes hispanos estimaba un 75\% de insatisfacción con la $\mathrm{IC}^{16}$, evidenciando la alta prevalencia de esta problemática.

Bajo este panorama se torna importante abonar aún más al estudio de la IC, en particular, en la población de jóvenes adolescentes, ya que las personas en esta etapa de la vida están consideradas de alto riesgo al ser más vulnerables a desarrollar mayor insatisfacción debido a los cambios físicos propios del desarrollo y a la presión sociocultural sobre la imagen del cuerpo en el contexto familiar y social en el que viven especialmente entre el círculo de amigos o por los medios de comunicación- ${ }^{1,17-19}$.

Sobre esto último, conviene reforzar la idea de que en estudios previos se ha evidenciado que la percepción de la imagen corporal está ligada a procesos socioculturales ${ }^{10}$, pero existe poca información sobre los factores individuales y de personalidad que intervienen en el mismo. El cuidar la imagen corporal puede tener diferentes significados personales desde los rasgos de personalidad; por ejemplo, quienes lo harían por controlar el peso, el tono muscular o razones de atractivo físico, difieren de quienes lo realizarían por motivos de salud, estado de ánimo o divertimento; asimismo, difiere en torno al sexo del sujeto ${ }^{20}$.

Finalmente, respecto de la personalidad, vale la pena referir en primer lugar que esta alude a una organización medianamente estable y duradera del carácter, temperamento, intelecto y físico de una persona que determina su adaptación específica al contexto, por lo que se puede señalar que son múltiples los factores que influyen en su desarrollo. ${ }^{21} \mathrm{Y}$ en segundo lugar, que actualmente existen pocos estudios que analicen los posibles vínculos de dicha dimensión psicológica con el tema de la Imagen Corporal ${ }^{20,22}$.

A partir de los referentes anteriores, el objetivo principal de este estudio consistió en identificar el nivel predictivo de las dimensiones o tipologías de la personalidad sobre la insatisfacción con la imagen corporal. Del mismo modo, se analizaron sus relaciones de forma bivariada y su comparación conforme al sexo de los participantes.

\section{MATERIALES Y MÉTODOS}

\section{Tipo de estudio}

El diseño de la investigación fue cuantitativo (dado que el análisis de datos es numérico), no experimental (ya que no se manipuló ninguna variable), de alcance predictivo (pues es posible predecir el comportamiento de la variable insatisfacción con la imagen corporal) y con una medición transversal (en razón de que se midió en una sola ocasión).

\section{Población y muestra}

La población estudiada estuvo conformada por estudiantes de un bachillerato tecnológico de 
un municipio de la región altos sur del estado de Jalisco (México). La muestra (no probabilística e intencional) se conformó por un total de 567 alumnos (279 hombres y 288 mujeres) con edades entre los 14 y 20 años $(M=16,29 ; \mathrm{DT}=$ $1,161)$. Como criterio de inclusión se consideró a los estudiantes registrados en el plantel y presentes al momento del levantamiento de la información. La institución educativa en la que se realizó este estudio cuenta con tres carreras de nivel técnico, a saber: Técnico Agropecuario, Técnico en Contabilidad y Técnico en Ofimática.

\section{Instrumentos}

El Cuestionario de Insatisfacción con la Imagen Corporal (Body Shape Questionnaire) ${ }^{23}$ está conformado por 34 ítems y tiene como objetivo explorar la autopercepción de la imagen corporal y detectar la presencia de insatisfacción con esta. A través de reactivos sobre aspectos de la vida cotidiana, apreciación, hábitos de salud y preocupaciones acerca del cuerpo, estima el nivel de insatisfacción con la forma corporal (p. ej., “¿Has evitado ir a actos sociales (por ejemplo a una fiesta) porque te has sentido mal respecto a tu figura?"). El cuestionario fue validado en población mexicana femenina y presenta un escalamiento tipo Likert de seis opciones de respuesta, que van de nunca a siempre ${ }^{24}$. El cuestionario mantiene índices de confiabilidad adecuados y, aunque algunos estudios señalan una estructura de dos factores, para esta investigación se tomó el resultado total del instrumento. El coeficiente de fiabilidad ( $\alpha$ de Cronbach) fue de 0,979 , mientras que para el presente estudio fue de 0,976 .

El Inventario de Personalidad NEO Revisado NEO FFI (versión reducida del NEO PI-R) ${ }^{25}$ es uno de los instrumentos más prestigiosos para la evaluación del constructo de interés. Su base teórica es el modelo de los Cinco Grandes factores de personalidad. Consta de 60 elemen- tos a los que se responde en una escala Likert de cinco opciones (de "En total desacuerdo" a "Totalmente de acuerdo") y permite la evaluación de cinco factores principales: neuroticismo, extraversión, apertura, amabilidad y responsabilidad. El alfa de Cronbach reportado en otros estudios oscila entre 0,89 y $0,91^{26}$ y en el presente fue de 0,71 .

Además de los instrumentos mencionados, se aplicó un cuestionario breve de datos sociodemográficos.

\section{Procedimiento}

De manera preliminar, se llevó a cabo una entrevista con las autoridades del bachillerato para la presentación, autorización y consentimiento del proyecto de investigación. El consentimiento informado se entregó a los alumnos con la finalidad de que fuera autorizado y firmado por los padres y devuelto a través de las autoridades del plantel. Una vez obtenidas las autorizaciones de los directivos de la institución y de los padres, se visitó, en sus respectivos grupos escolares, a los alumnos que cursan las carreras antes señaladas y se les pidió que contestaran los instrumentos referidos.

\section{Declaración sobre aspectos éticos}

El estudio se integra dentro del rango de riesgo mínimo según las normas éticas de la investigación con seres humanos, garantizando los derechos y favoreciendo el bienestar de cada participante. Asimismo, durante el proceso de recolección de datos se administró un consentimiento informado tanto a los estudiantes como a los padres de familia cuyos hijos eran menores de edad. En dicho documento los investigadores se comprometieron a no divulgar los datos de manera individual, sino globales y solo con propósitos académicos y científicos. Cabe señalar que durante la aplicación del instrumento se explicaron de forma detallada los objetivos de 
Juan Francisco Caldera-Montes, Oscar Ulises Reynoso-González, Diana Nuño-Camarena, Iván Alejandro Caldera-Zamora, Ignacio Pérez-Púlido, Claudia Arcelia Gómez-Álvarez

la investigación a los estudiantes, profesores y directivos de la institución educativa.

\section{Análisis estadístico}

Se realizaron análisis descriptivos y bivariados para observar el comportamiento de las variables Insatisfacción con la Imagen corporal y la Personalidad (con sus respectivos factores o dimensiones). Posteriormente se realizó una regresión lineal múltiple a través del método por pasos (stepwise) con el objetivo de identificar los factores que explican y predicen la ocurrencia de la "Insatisfacción de la IC" en los estudiantes de bachillerato. El análisis de los datos se realizó con el programa estadístico SPSS, versión 22.

\section{RESULTADOS}

De forma inicial se calcularon las medias y desviaciones estándar de las variables Insatisfacción con la imagen corporal y Personalidad junto a sus respectivos factores. Para su presentación, se muestran los resultados generales y por sexo (Tabla 1).

Tabla 1.Medias, Desviaciones estándar y Totales de Mujeres y Hombres.

\begin{tabular}{|l|c|c|c|c|c|c|}
\hline \multirow{2}{*}{\multicolumn{1}{|c|}{ Variable/factor }} & \multicolumn{2}{c|}{ Total } & \multicolumn{2}{c|}{ Mujeres } & \multicolumn{2}{c|}{ Hombres } \\
\cline { 2 - 7 } & Media & Desv. std. & Media & Desv. std. & Media & Desv. std. \\
\hline $\begin{array}{l}\text { INSATISFACCIÓN CON LA } \\
\text { IMAGEN CORPORAL }\end{array}$ & 60,01 & 31,888 & 69,61 & 36,084 & 50,11 & 23,071 \\
\hline NEUROTICISMO & 19,14 & 6,887 & 20,20 & 6,682 & 18,05 & 6,936 \\
\hline EXTROVERSIÓN & 29,03 & 6,902 & 29,42 & 6,996 & 28,62 & 6,792 \\
\hline APERTURA & 26,60 & 5,628 & 26,95 & 5,563 & 26,24 & 5,682 \\
\hline AMABILIDAD & 28,20 & 5,694 & 28,72 & 5,829 & 27,66 & 5,510 \\
\hline RESPONSABILIDAD & 28,57 & 5,904 & 28,57 & 5,560 & 28,57 & 6,249 \\
\hline
\end{tabular}

Enseguida se calcularon las frecuencias absolutas y relativas de los niveles de Insatisfacción con la imagen corporal de los estudiantes (Tabla 2). Cabe resaltar el hecho de que, bajo esta clasificación, 153 estudiantes obtuvieron niveles medios y altos.

Tabla 2. Frecuencias por nivel de Insatisfacción de la imagen corporal.

\begin{tabular}{|c|c|c|c|c|c|}
\hline \multicolumn{2}{|c|}{ Baja } & \multicolumn{2}{c|}{ Media } & \multicolumn{2}{c|}{ Alta } \\
\hline $\mathrm{N}$ & $\%$ & $\mathrm{~N}$ & $\%$ & $\mathrm{~N}$ & $\%$ \\
\hline 414 & 73,0 & 147 & 25,9 & 6 & 1,1 \\
\hline
\end{tabular}

Posteriormente, con el objetivo de detectar diferencias significativas entre hombres y mujeres, se realizaron un conjunto de pruebas U de Mann Whitney (considerando la ausencia de normalidad en la distribución de las variables y factores). Los resultados de la prueba se muestran en la Tabla 3.

Como se puede apreciar en la tabla anterior, se encontraron diferencias significativas en la variable "Insatisfacción con la imagen corporal" $(\mathrm{Z}=-8,411 ; \mathrm{p}=0,000)$ y en los factores de la personalidad "Neuroticismo" ( $Z=-3,242-; p=0,001)$, "Apertura" $(\mathrm{Z}=--2,387 ; \mathrm{p}=0,017)$ y "Amabilidad" 
$(\mathrm{Z}=-2,415 ; \mathrm{p}=0,016)$. En todos los casos las mujeres obtuvieron puntuaciones más altas en relación con los hombres.

Tabla 3. Comparación entre sexo.

\begin{tabular}{|l|c|c|}
\hline \multicolumn{1}{|c|}{ Variable/factor } & $\mathbf{Z}$ & Significancia $(\mathbf{p})$ \\
\hline $\begin{array}{l}\text { INSATISFACCIÓN } \\
\text { CON LA IMAGEN } \\
\text { CORPORAL }\end{array}$ & $-8,411$ & $0,000^{*}$ \\
\hline NEUROTICISMO & $-3,242$ & $0,001^{\star}$ \\
\hline EXTROVERSIÓN & $-1,291$ & 0,197 \\
\hline APERTURA & $-2,387$ & $0,017^{*}$ \\
\hline AMABILIDAD & $-2,415$ & $0,016^{*}$ \\
\hline RESPONSABILIDAD & $-0,072$ & 0,942 \\
\hline
\end{tabular}

${ }^{*} \mathrm{p}<0,05$

A continuación, con la intención de identificar posibles correlaciones entre la variable Insatisfacción con la imagen corporal y los distintos factores de la prueba de Personalidad, se utilizó la prueba Rho de Spearman (Tabla 4).
Como se observa, existen correlaciones significativas entre la insatisfacción con la imagen corporal y el factor "Neuroticismo" (positiva y media), "Extroversión" (Negativa y muy baja), "Amabilidad" (negativa y muy baja) y "Responsabilidad (negativa y muy baja).

Por último, se realizó un análisis de regresión lineal múltiple a través del método por pasos, considerando a la "Insatisfacción con la imagen corporal" como variable dependiente y a los factores "Neuroticismo", "Extroversión", "Amabilidad" y "Responsabilidad" como independientes. Además, teniendo en cuenta que en el caso de la Insatisfacción con la imagen corporal el contraste de la variable "sexo" mostró diferencias significativas, se optó por integrar la variable en el proceso con la denominación de ficticia o "dummy". Los resultados indicaron que el segundo modelo presentaba las mejores propiedades sin corromper ninguno de los supuestos estadísticos de la prueba. El análisis mostró un valor de regresión corregido medio-bajo $\left(\mathrm{R}^{2}=0,219\right)$, es decir, que su valor de predicción es de 21,9\% (Tabla 5).

Tabla 4. Correlaciones entre la insatisfacción de la imagen corporal y los factores de la personalidad.

\begin{tabular}{|l|c|c|c|c|c|}
\hline & Neuroticismo & Extroversión & Apertura & Amabilidad & Responsabilidad \\
\hline $\begin{array}{l}\text { Insatisfacción con la } \\
\text { imagen corporal }\end{array}$ & $0,433^{*}$ & $-0,131^{\star}$ & 0,004 & $-0,149^{\star}$ & $-0,134^{*}$ \\
\hline $\mathrm{p}<0,01$ &
\end{tabular}

Tabla 5. Modelo de regresión múltiple (por pasos).

\begin{tabular}{|l|c|c|c|c|l|c|c|}
\hline & $\mathbf{r}$ & $\mathbf{R}^{2}$ & $\mathbf{R}^{2}$ ajustado & Error típ. & Durbin-Watson & $\mathbf{f}$ & Sig. \\
\hline $\begin{array}{l}\text { Insatisfacción con la } \\
\text { imagen corporal }\end{array}$ & 0,470 & 0,221 & 0,219 & 28,189 & 1,989 & 80,165 & 0,000 \\
\hline
\end{tabular}


Juan Francisco Caldera-Montes, Oscar Ulises Reynoso-González, Diana Nuño-Camarena, Iván Alejandro Caldera-Zamora, Ignacio Pérez-Púlido, Claudia Arcelia Gómez-Álvarez

Los coeficientes obtenidos presentan estadísticos de no colinealidad aceptables (valores menores de 15 en los índices de condición), junto a la independencia en los residuos (DW=1,989). Del mismo modo, el gráfico de residuos estandarizados y pronósticos tipificados confirmó la normalidad y homogeneidad de los residuos. Los resultados muestran el rasgo o dimensión de la personalidad neurótica $(\beta=0,362)$ como el factor de mayor importancia para la insatisfacción de la imagen corporal, seguido del sexo $(\beta=0,250)$, específicamente el caso de las mujeres (Tabla 6).

Tabla 6. Coeficientes de regresión de la variable "Insatisfacción con la Imagen Corporal".

\begin{tabular}{|c|c|c|c|c|c|c|c|}
\hline & \multicolumn{2}{|c|}{$\begin{array}{l}\text { Coeficientes no } \\
\text { estandarizados }\end{array}$} & \multirow{2}{*}{$\begin{array}{c}\begin{array}{c}\text { Coeficientes } \\
\text { estandarizados }\end{array} \\
\text { Beta }(\beta)\end{array}$} & \multirow{2}{*}{$\mathbf{t}$} & \multirow{2}{*}{ Sig. } & \multicolumn{2}{|c|}{ Estadísticas de colinealidad } \\
\hline & B & Error est. & & & & Tolerancia & VIF \\
\hline (Constante) & 3,965 & 4.672 & & 0,849 & 0,396 & & \\
\hline Neuroticismo & 1,675 & 0,174 & 0,362 & 9,618 & 0,000 & 0,976 & 1,025 \\
\hline Sexo & 15,902 & 2,397 & 0,250 & 6,633 & 0,000 & 0,976 & 1,025 \\
\hline Ecuación & $\begin{array}{l}\text { Insatisfa } \\
\text { el caso d }\end{array}$ & $\begin{array}{l}\text { on con la Ir } \\
\text { s mujeres) }\end{array}$ & gen corpor & $65+$ & 75) & (ismo) $]+$ & (sólo en \\
\hline
\end{tabular}

\section{DISCUSIÓN}

A partir de los resultados expuestos, se pudo advertir que, por lo general, los alumnos presentan puntajes bajos (considerando que la media teórica del instrumento es de 119) de insatisfacción con la imagen corporal. Sin embargo, el hecho de que al menos 153 jóvenes (27\%) se encuentren en los niveles medio y alto de insatisfacción llama la atención sobre todo al considerar que se conoce que tal condición correlaciona con aspectos graves como la depresión ${ }^{4}$, los trastornos de la conducta alimentaria y la ideación o intento suicida ${ }^{3}$. Las causas que pueden esgrimirse respecto de dicha frecuencia pueden deberse a los múltiples cambios experimentados por la adolescencia, tanto físicos como psicológicos, en los cuales se tiende a buscar una aceptación sociocultural sobre la imagen de su cuerpo ${ }^{5}$.

En cuanto a los puntajes totales en la prueba que se sustenta en los cinco factores de la personalidad, se advierte que los alumnos se ubican en el nivel alto en neuroticismo, lo cual quiere decir que se encuentran en tendencia de experimentar estados emocionales negativos, es decir, presentan susceptibilidad a perturbaciones psicológicas ${ }^{27}$; tienen un nivel bajo de extroversión: los alumnos en términos generales no tienden a llamar la atención de sus pares; presentan niveles bajos de apertura, lo cual indica personalidades convencionales y conservadoras ${ }^{27}$; manifiestan un nivel bajo de amabilidad; y por último, se evidencia que el nivel de responsabilidad es bajo, se encontró que los alumnos son menos rigurosos en cuestión de principios morales.

En lo que respecta a comparaciones entre hombres y mujeres en relación con la IC, se encontró que existen diferencias significativas, obteniendo mayores puntajes las mujeres. Tal situación concuerda plenamente con diversos estudios que abordan el tema y se explica debido a lo dicho por Trejo, et al ${ }^{1}$ en el sentido de que la cultura de la imagen corporal suele generar mayores presiones sociales en las mujeres que en los varones. Tal condición se refuerza todavía más debido a las características socioculturales de la región en 
donde se realizó el estudio, la cual se caracteriza por un fuerte culto a la belleza femenina ${ }^{14}$.

En cuestión de neuroticismo, las mujeres también reportan mayores puntajes que los hombres, lo mismo sucede con la variable de amabilidad. Lo anterior da a entender que las féminas son personas con tendencias más ansiosas, impulsivas y vulnerables, pero a su vez, muestran mayor amabilidad ${ }^{27}$. Dichos hallazgos pueden explicarse a partir de considerar que los estilos de crianza en hombres y mujeres son distintos en prácticamente todas las sociedades humanas y de manera muy marcada en la Región Altos Sur de Jalisco, en donde prevalecen valores conocidos como tradicionales o patriarcales. Por lo que toca a las variables de extroversión, apertura y responsabilidad, no se encuentran diferencias significativas. Esto quiere decir que tanto los hombres como las mujeres suelen ser extrovertidos, muestran apertura y son responsables en igual medida.

Por su parte, la relación significativa y positiva entre la insatisfacción con la imagen corporal y la personalidad neurótica, refuerza lo encontrado por otras investigaciones como la de Castro et $a l^{21}$. Tal asociación se puede explicar a partir del hecho de que la personalidad tiende a relacionarse con susceptibilidad psicológica, lo que da a entender que las personas con puntajes altos en esta dimensión de la personalidad pueden ser proclives a trastornos de percepción y, por ello, a insatisfacción con la imagen corporal ${ }^{27}$.

Con relación a la asociación significativa y negativa entre el factor de la personalidad "amabilidad" y la insatisfacción con la imagen corporal, se puede argumentar que dicha condición existe debido a que las personas poco amables se distinguen por presentar rasgos como ser competitivos, irritables, egoístas y críticos, los cuales son característicos de una alta tendencia a la comparación social ${ }^{28}$.
Por otro lado, la relación significativa y negativa entre el factor de la personalidad "responsabilidad" y la insatisfacción con la imagen corporal se puede deber al hecho de que las personas poco organizadas, metódicas y meticulosas (menos responsables) tienden a ser impulsivas $y$, por tanto, emocionalmente más susceptibles a lo que los demás puedan decir o pensar sobre ellos, especialmente sobre su apariencia y forma corporal. Sin embargo, dicha afirmación debe tomarse con cautela debido al bajo nivel de correlación y, sobre todo, es necesario realizar más estudios que confirmen el vínculo entre dichas variables o dimensiones.

En cuanto al modelo predictivo generado a través del análisis de regresión múltiple se constató que la insatisfacción con la imagen corporal puede predecirse con un $21,9 \%$ de probabilidades a partir de la dimensión de la personalidad "neuroticismo" y del sexo "mujer", condición que implica que las féminas cuyos rasgos "neuróticos" son preeminentes son más susceptibles de presentar insatisfacción con la imagen corporal, y por tanto que dicha condición provoque malestar psicológico. Al respecto, cabe señalar que tal afirmación coincide con lo evidenciado por lo descrito en la literatura de investigación en esta materia ${ }^{2,3,8}$. Pero sobre todo, la importancia que tienen dichas dimensiones o variables en la prensencia de la IC insatisfecha, en particular, si se toma en cuenta que las mismas son además típicas de los trastornos de conducta alimentaria con los que la IC está fuertemente relacionada ${ }^{13,29,30}$.

Sin embargo, y a pesar de lo afirmado en el párrafo anterior, es evidente que dichos aspectos no son suficientes para predecir o explicar la presencia del trastorno estudiado, por lo que valdría la pena efectuar estudios posteriores que incluyan otras características que pudieran revelar en mayor medida la presencia de la insatis- 
Juan Francisco Caldera-Montes, Oscar Ulises Reynoso-González, Diana Nuño-Camarena, Iván Alejandro Caldera-Zamora, Ignacio Pérez-Púlido, Claudia Arcelia Gómez-Álvarez

facción de la imagen corporal en adolescentes, por ejemplo, la autoestima, la inteligencia emocional, la conducta alimentaria, la práctica del ejercicio o la autoeficacia, entre otras.

Finalmente, en cuanto a las limitaciones de esta investigación, es importante señalar que los resultados obtenidos emergen de autoinformes, situación que implica que las respuestas pueden estar sesgadas al ser el propio participante quien informa de sus conductas y actitudes. Por tanto, convendría, en estudios posteriores, utilizar otras estrategias de investigación, como por ejemplo, modelos de ecuaciones estructurales, diseños experimentales o enfoques de investigación de corte cualitativo.

\section{DECLARACIÓN SOBRE CONFLICTOS DE INTERESES}

Los autores manifestamos que somos independientes y que no se contó con apoyo económico de ninguna empresa o institución para la planeación, ejecución y publicación de este proyecto de investigación. Por tal motivo, declaramos no tener conflicto de intereses.

\section{REFERENCIAS BIBLIOGRÁFICAS}

1. Trejo P, Mollinedo F, Araujo R, Valdez G, Sánchez M. Hábitos de actividad física y cánones de imagen corporal en estudiantes universitarios. Revista Cubana de Medicina Integral. 2016 Ene; 32(1): 72-82. Disponible en: http://scielo.sld.cu/ pdf/mgi/v32n1/mgi09116.pdf

2. Aceves J, García S, González A. Insatisfacción de la imagen corporal y autopercepción en adolescentes de una escuela secundaria. Neurología, Neurocirugía y Psiquiatría. 2011
Sep; 44(4): 128-132. Disponible en: http://www. medigraphic.com/pdfs/revneuneupsi/nnp2011/nnp114d.pdf

3. Benel R, Campos S, Cruzado L. Insatisfacción corporal en estudiantes de medicina de la Universidad Peruana Cayetano Heredia medida con el Body Shape Questionnaire. Rev Neuropsiquiatr 75. 2012 Sep; 75(3): 85-92. Disponible en: http://www.upch.edu.pe/ vrinve/dugic/revistas/index.php/RNP/article/ viewFile/1226/1258

4. Griffoulière M, Rivarola M, Galarsi M, Penna F. Imagen corporal y estrategias de afrontamiento en adolescentes escolarizados, de la ciudad de San Luis, Argentina. Fundamentos En Humanidades. 2014 Ene; 27(1): 95-117. Disponible en: http:// fundamentos.unsl.edu.ar/pdf/articulo-27-95.pdf

5. Fernández J, González I, Contreras O, Cuevas R. Relación entre imagen corporal $\mathrm{y}$ autoconcepto físico en mujeres adolescentes. Revista latinoamericana de psicología. 2015 Ene; 47(1): 25-33. Disponible en: DOI: 10.1016/ S0120-0534(15)30003-0

6. Neves V, Soares A, Sousa L, Aparecida M, Caputo M. Estudo transversal sobre imagem corporal de adolescentes. Psicologia: teoria e prática. 2013 Sep; 15(3): 151-162. Disponible en: http://pepsic. bvsalud.org/pdf/ptp/v15n3/11.pdf

7. Bobadilla S, López A. Distorsión de imagen corporal percibida e imaginada. un posible factor para la obesidad y el sobrepeso en mexicanos. Rev Med Inst Mex Seguro Soc. 2014 Oct; 52(4): 408414. Disponible en: http://www.medigraphic. com/pdfs/imss/im-2014/im144m.pdf

8. Meza C, Pompa E. Insatisfacción con la imagen corporal en adolescentes de Monterrey. Daena: International Journal of Good Conscience. 2013 Ene; 8(1): 32-43. Disponible en: http://www. spentamexico.org/v8-n1/A4.8(1)32-43.pdf 
9. Hernández A, Mancilla J, Álvarez G, Ortega M, Larios M, Martínez I. Edad, consciencia e interiorización del ideal corporal como predictores de insatisfacción y conductas alimentarias anómalas. Revista Colombiana de Psicología. 2013 Ene; 22(1): 121-133. Disponible: https://revistas.unal.edu.co/index. php/psicologia/article/view/28326/43288

10. Vaquero R, Alacid F, Muyor J, López P. Imagen corporal; revisión bibliográfica. Nutrición Hospitalaria. 2013 Ene; 28(1): 27-35. Disponible en: DOI:10.3305/nh.2013.28.1.6016

11. Gimenez P, Correché M, Rivarola M. Autoestima e Imagen Corporal. Estrategias de intervención psicológica para mejorar el bienestar psicológico en pre- adolescentes en una escuela de la ciudad de San Luis, Argentina. Fundamentos en Humanidades. 2013 Ene; 14(1): 83-93. Disponible en: http://fundamentos.unsl.edu.ar/ pdf/articulo-27-83.pdf

12. Guest-Jelley A, Klein M. Yoga e imagen corporal México: Paidós; 2016.

13. Anuel A, Bracho A, Brito N, Rondón J, Sulbarán D. Autoaceptación y mecanismos cognitivos sobre la imagen corporal. Psicothema. 2012 Sep; 24(3): 390-395. Disponible em: http://www. psicothema.com/pdf/4028.pdf

14. Gómez A, Sánchez B, Mahedero M. Insatisfacción y distorsión de la imagen corporal en adolescentes de doce a diecisiete años de edad. 2013 Ene; 15(1): 54-63. Disponible en: http://agora-revista.blogs.uva.es/files/2013/07/ agora_15_1d_gomez_et_al.pdf

15. Acosta M, Gómez G. Insatisfacción corporal y seguimiento de dieta. Una comparación transcultural entre adolescentes de España y México. International Journal of Clinical and Health Psychology. 2003 Ene; 3(1): 9-21. Disponible en: http://aepc.es/ijchp/articulos_ pdf/ijchp-57.pdf
16. Rodríguez S, Cruz S. Insatisfacción corporal en adolescentes latinoamericanas y españolas. Psicothema. 2008 Ene; 20(1): 131-137. Disponible en: http://www.psicothema.com/PDF/3438.pdf

17. Soto M, Marín B, Aguinaga I, Guillén F, Serrano I, Canga N, et al. Análisis de la percepción de la imagen corporal que tienen los estudiantes universitarios de Navarra. Nutrición Hospitalaria. 2015 May; 31(5): 22692275. Disponible en: http://www.redalyc.org/ pdf/3092/309238514048.pdf

18. Gonçalves S, Bedin L. Bienestar, salud e imagen corporal de adolescentes brasileiros: la importancia de los contextos familiar, de amistad y escolar. Universitas Psychologica. 2016 Sep; 14(4): 1300-1410. Disponible en: DOI:10.11144/ Javeriana.up14-4.bsic

19. Cruz M, Salaberria K, Rodríguez S, Echeburúa E. Imagen corporal y realización de dieta: diferencias entre adolescentes españolas y latinoamericanas. 2012 Jul; 12(3): 699-708. Disponible en DOI: 10.11144/Javeriana.UPSY12-3.icrd

20. Baile J, González A, Ramírez C, Suárez P. Imagen corporal, hábitos alimentarios y hábitos de ejercicio físico en hombres usuarios de gimnasio y hombres universitarios no usuarios. Revista de psicología del deporte. 2011 Abr; 20(2): 353366. Disponible en: http://www.rpd-online.com/ article/view/783/774

21. Castro R, Molero D, Cachón J, Zagalaz M. Factores de la personalidad y físicoculturismo: Indicadores asociados a la vigorexia. Revista de Psicología del Deporte. 2014 Abr; 23(2): 295-300. Disponible en: http://www.rpd-online.com/article/view/ v23-n2-castro-lopez-molero-etal/pdf_es

22. Gázquez J, Pérez M, Mercader I, Molero $M$, Inglés C. Repercusión del optimismo y de los cinco grandes factores de la personalidad sobre la salud de personas mayores. Universitas Psychologica. 2014 Sep; 13(3): 15-23. Disponible en: DOI: 10.11144/Javeriana.UPSY13-3.rocg 
23. Cooper P, Taylor M, Cooper Z, Fairburn C. The development and validation of the Body Shape Questionnaire. International Journal of Eating Disorders. 1987 Oct; 6(4): 485-494. Disponible en: DOI: $10.1002 / 1098-108 X(198707)$

24. Vázquez R, Galán J, López X, Álvarez G, Mancilla J, Caballero A, et. Al. Validez del Body Shape Questionnaire (BSQ) en Mujeres Mexicanas. Revista Mexicana de Trastornos Alimentarios. 2011 Ene; 2(1): 42-52. Disponible en: http:// www.scielo.org.mx/pdf/rmta/v2n1/v2n1a5.pdf

25. Costa P, MaCrae R. The revised NEO personality Inventory (NEO PI-R) and $\mathrm{NEO}$ five-factor Inventory (NEO-FFI) professional manual FL: Psychological Assessment Resources: Odessa.; 1987.

26. Aguilar M, Landa J. Relación entre inteligencia emocional percibida, personalidad y capacidad empática de enfermería. Behavioral Psychology. 2009 Jun; 17(2): 351-364. Disponible en: https:// www.thefreelibrary.com/

27. Salvador J, Sánchez M. Sistema experto: Rasgos de la personalidad; 2008. Disponible en: http://studylib.es/doc/6024697/sistemaexperto--rasgos-de-la-personalidad
28. Buunk A, Belmonte J, Peiró J, Zurriaga R, Gibbons F. Diferencias individuales en la comparación social: propiedades de la escala española de orientación hacia la comparación social. Revista Latinoamericana de Psicología. 2005 Sep; 37(3): 561-579. Disponible en: http://www.redalyc.org/ articulo.oa?id $=80537308$

29. Altamirano M, Vizmanos B, Unikel C. Continuo de conductas alimentarias de riesgo en adolescentes de México. Revista Panamericana de Salud Pública. 2011 Oct; 30(5): 401-407. Disponible en: https://pdfs.semanticscholar.org/38a3/790e2eab5 7d185382eb99ca77909176faecd.pdf

30. González R, García P, Martínez J. Valoración de la Imagen Corporal y de los comportamientos alimentarios en universitarios. Revista de Psicopatología y Psicología Clínica. 2013 Ene; 18(1): 45-49. Disponible en: http://e-spacio. uned.es/fez/eserv.php?pid=bibliuned:Psicopat2013-18-1-6015\&dsID=Documento.pdf. 